\title{
Location Analysis of Electric Vehicle Charging Stations for Maximum Capacity and Coverage
}

\author{
I. Safak Bayram \\ Department of Electronic and Electrical Engineering \\ University of Strathclyde \\ Glasgow, United Kingdom, G1 1XQ \\ safak.bayram@strath.ac.uk
}

\author{
Sertac Bayhan \\ Qatar Environment and Energy Research Institute, \\ Hamad Bin Khalifa University \\ PO Box: 34110 Doha - Qatar \\ sbayhan@hbku.edu.qa
}

\begin{abstract}
Electric vehicle charging facility location is a critical component of long-term strategic planning. Integration of electric vehicles into mainstream adoption has unique characteristics as it requires a careful investigation of both electric and transportation networks. In this paper, we provide an overview of recent approaches in location analyses of electric vehicle charging infrastructures. We review approaches from classical operations research for fast and slow charging stations. Sample formulations along with case studies are presented to provide insights. We discuss that classical methods are appropriate to address the coverage of charging networks which is defined as average time or distance to reach a charging station when needed. On the other hand, calculating required capacity, defined as the individual charging resources at each node, is still an open research topic. In the final part, we present stochastic facility location theory that uses queuing and other probabilistic approaches.

Index Terms-location theory, electric vehicle charging stations, transportation networks, power grids
\end{abstract}

\section{INTRODUCTION}

To meet net-zero carbon emission goals, there has been a global push towards mass-adoption of plug-in electric vehicles (PEV). In line with such environmental goals, various countries including the United Kingdom, France, Norway, and India have announced a ban on the sale of new diesel vehicles by 2040 onwards [1]. On the other hand, widespread PEV adoption requires supporting charging infrastructure to provide necessary coverage and capacity to fuel a large numbers of trips comparable to gas station networks. To that end, this paper presents a literature overview of charging station location analyses to spatio-temporal electric vehicle demand.

In order for a fast transport-electrification, the supporting charging infrastructures need to compete with well-established supply chain practices of petroleum downstream industry. The primary differences between electric transportation and the existing carbon-based are on the storage and delivery of the supply. Power grids are real-time supply demand systems with limited storage capacities and tight control on supply-side. Due to the increasing shares of variable renewable generation, power grids are becoming more stressed, hence, the transition towards electric mobility requires a careful investigation of

This publication was made possible by NPRP12S-0214-190083 from the Qatar National Research Fund (a member of Qatar Foundation). The statements made herein are solely the responsibility of the authors.
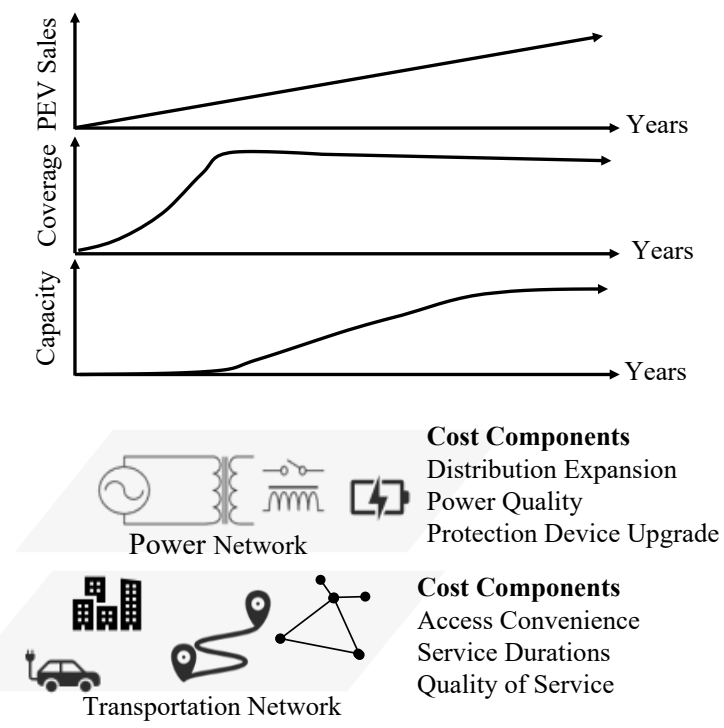

Fig. 1: Overview of charging network capacity and coverage needs.

potential disruptive impacts of PEV chargings [2]. Previous research shows that even small clusters of simultaneous PEV charging could lead to grid problems such as premature aging of distribution transformers [3], power quality deterioration [4], shortages [5], and possibly increase peak system demand. Therefore, laying the foundations for sustainable charging infrastructures is a critical step for a seamless integration of PEVs [2]. On the other hand, there is a century-old industry on the delivery and storage of petroleum products for transportation systems supporting millions of gasoline vehicles worldwide.

PEV charging facility deployment is a complex long-term planning that has two key components, coverage and capacity. The first one is described as the ability of PEVs to find a charging node within a certain time and travel duration. Widecoverage of charging services is critical to boost PEV sales. On the other hand, as PEV sales grow, there is a need to increase charging capacity by deploying additional chargers and upgrading network elements. Charging nodes may require hosting different charging types [6] or use on-site storage units to meet PEV demand [7]. Moreover, decision making 
involves both power and transportation networks and different cost components that have short and long-term impacts. An overview of charging station siting problem is depicted in Fig. 1.

Furthermore, mass PEV adoption requires addressing a chicken-egg problem: the PEV sales are linked to the convenient access to charging nodes (the chicken problem), thus more charging service is needed. On the other hand, business owners are not likely to expand charging networks if the penetration rates do not offer profitability (the egg problem). Moreover, the location of charging facilities will impact electricity consumption, and power grid operations. Hence, different from any other facility location problem, the proper deployment of PEV stations has impacts on environment, electricity, and petroleum industries.

In general, location theory aims to find an appropriate location for facilities to optimise single or multiple quantifiable objectives related to customer needs, power grid constraints, associated capital cost, and environmental goals such as lowemission zones in city centres [8]. Although, the literature on facility theory contains a rich body of literature [9]-[14], the applications for PEV charging location is sparse and requires further investigation [15]-[20]. The primary reason is that the electrification rates are still low (e.g., less than 5\% of all cars) and the real-world challenges on vehicle, driver, and charging infrastructure are still unknown. In this paper, we first explain classical facility location problems, approaches, and applications. Next, we explain unique characteristics of charging station problems and differentiate slow and fast charging facility location problems. Moreover, we discuss probabilistic approaches and conclude the paper with multidisciplinary approaches.

\section{Facility Location Problems: Classical APPROACHES}

\section{A. Background}

The facility location problems were first addressed by the pioneering studies of Alfred Weber in 1909, in which the location problem of warehouses is considered to minimise the distance between the customers and the facility. Later, Alfred Hotelling studied facility location of two facilities on a line model [12]. In this case, customers are assumed to be uniformly distributed along a line and they prefer to go to the nearest facility to minimise travel cost. Hence, the optimal facility locations for both facilities are readily found to be at the middle points.

The interest in facility location problems flourished during mid-1960s in parallel to the expansion of the telecommunication networks. In such networks, the main goal was decide on the optimal locations of telephone switching facilities to minimise the cost associated with the infrastructure. Over the next consecutive years, researchers addressed a number of private and public sector facility problems. In the private sector, location problems considers banks/ATMs, industrial plants, and retail facilities. In the public sector, urban operations research focuses on locating emergency vehicles, schools, hospitals, and fire stations [9]-[12]. It is noteworthy that the private sector priorities minimisation of monetary cost, while public sector aims to optimise access time by the public. A similar dichotomy arises in the context of charging station deployment. The currently operational stations are owned by the private sector, while they are, at the same time, incentivised by the governments. Thus, location problems may need to consider competing, and even perhaps opposing objectives, where one tends to reduce the siting cost and another one to improve accessibility.

\section{B. Design Objectives}

Charging station facility location problems are capitalintensive onetime decisions that have long-lasting ramifications. We differentiate charging station location problems into two groups as efforts for fast and slow charging stations. Fast chargers are designed to mimic gas stations and designed to host a few vehicles (e.g., 5-10) at the same time. On the other hand, slow charging stations, typically composed of level 2 chargers, are located at parking lots of shopping malls, universities, hospitals, and airports. For the case of fast (50 $\mathrm{kW})$ and ultra-fast $(>50 \mathrm{~kW})$ charging stations [21], the high cost of electrical equipments, energy storage systems, and land acquisition make decision-making of such facilities a longterm investment. Moreover, station location may need to incorporate future changes in the vehicle distribution, driving routes between the points of interests, and changes in population profiles. On the other hand, locating slow-charging stations is a relatively less complicated problem since chargers will be deployed at existing public spaces.

Location analyses of charging stations involves the following components; (1) density of PEVs in a given geographical area; (2) the charging facilities/types to be installed; (3) a space in which stations are located; and (4) a metric that measures the distances or commute time between stations and the PEVs. Such analyses need using proper models for facility locations and address the following questions:

1) Where should each station be located in a given wellconfined region?

2) How many charging facilities should be located to guarantee a pre-determined quality of service goals?

3) What should be the capacity of each charging station?

4) What type of chargers should be chosen?

5) Should the charging facilities be sited as clusters or sets of interconnected regions?

Moreover, additional challenges might emerge due to impending developments such as resource planning based on renewables and storage units at each station. Addressing these research challenges depend on the context in which station locations is formulated and solved. For example, in urban city centres, city planners may prefer installing fast-charging facilities as users may not have access to parking lots for long hours. However, the availability of physical land in small towns makes slow-charging facilities more attractive, as the driving ranges of the daily commutes are short. A comparison of slow and fast charging stations are presented in Table I. 
TABLE I: Characteristics of slow and fast charging station location problems.

\begin{tabular}{lll}
\hline & Slow Charging & Fast Charging \\
\hline Single Charger Power & $6-12 \mathrm{~kW}$ & $50+\mathrm{kW}$ \\
Typical Station Size & $20-100$ chargers & $2-10$ chargers \\
Service Duration & 3-8 hours & $20-30$ min \\
Vehicle Status & Parked & Mobile \\
Candidate Locations & Discrete - parking lots & Continuous- anywhere \\
Relevant Theory & Discrete location (Fig 3) & Flow Capturing \\
\hline
\end{tabular}

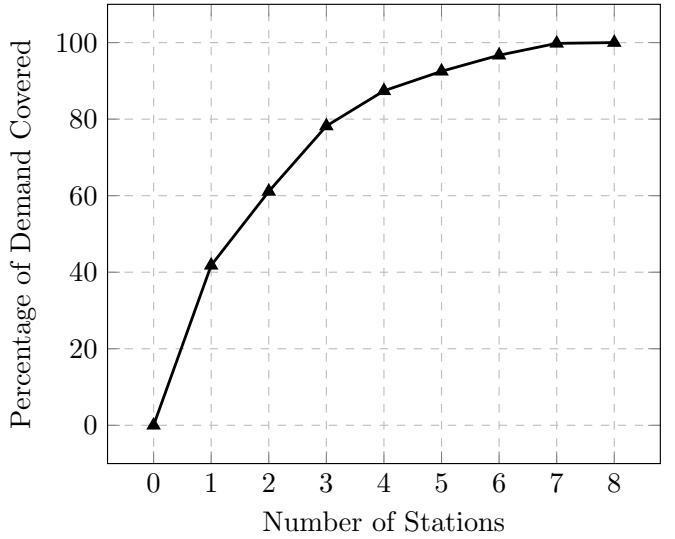

Fig. 2: Facility coverage trade-off for basic coverage problem. Data set is presented in [22].

\section{Discrete Network Models for Slow Charging Location}

Classical facility location problems aim to provide a certain level of coverage for a given set of demand nodes in a network. Since, customer demand is typically unevenly distributed, increasing the number of facilities does not necessarily bring the same level of coverage. We provide a sample case study to clarify the matters. We consider a case where we want to deploy charging stations in a given city such that any vehicle can drive to a charger under $D$ miles. Hence, the objective is to minimise the number of chargers needed so that all customers can reach a station within the D miles. This kind of problems are studied under set covering problems [22]. Even though deploying minimum number of chargers to cover entire customer demand may help to beat range anxiety, a number of charging nodes may be under-utilised due to uneven demand. Assume that locating $N$ charging stations satisfies the $D$ miles requirement. Then, the penalty for closing one station, that is the network will be not serving a portion of the load, is considerably lower than than $1 / N$ [22]. Hence, an alternative approach would be to maximise demand that can be served with a given number of stations. In location theory, this problem is known as the Maximum Covering Problem and an example is presented in Fig. 2 adopted from [22]. In this case study, eight charging stations are needed to serve $100 \%$ customer demand. If the station operator decides to sacrifice small $1-2 \%$ of the demand, then the deployment cost reduces about one eighth. The third location model assumes that the

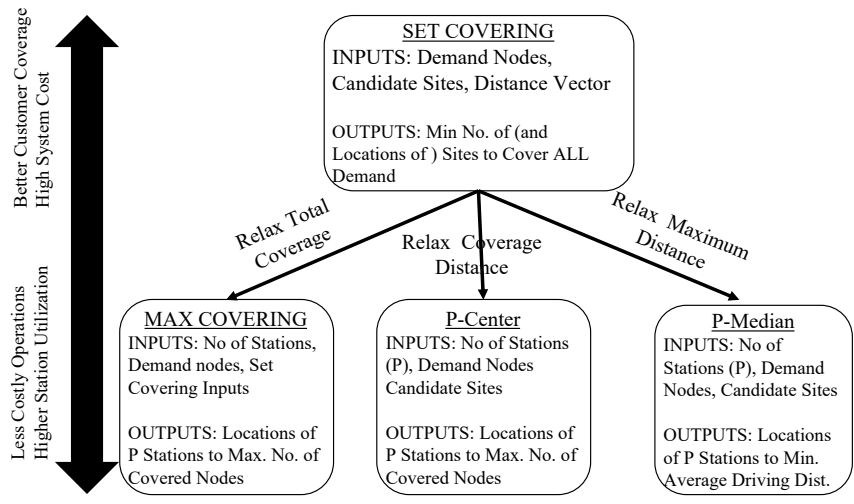

Fig. 3: Discrete location problems.

station operator has a fixed budget and aims to minimise the maximum driving distance with $P$ stations (referred as $P$ center problem). A sample formulation for $P$-center problem is presented next. The goal is to minimise the maximum distance $d$. Let $N$ represent the set of nodes, $\mathcal{S} \subset \mathcal{N}$ is the set of potential sites for charging facilities. We further employ a binary decision variable $b_{p}$, which is equal to one if site $p$ is selected, and set to zero otherwise. The problem can be formulated as below,

$$
\begin{array}{ll}
\operatorname{minimize} & d \\
\text { s.t. } & d-\sum_{p \in S} w_{n} d_{n} a_{n p} \geq 0 \forall n \in \mathcal{N} \\
& \sum_{p \in S} a_{n p}=1, \forall n \in \mathcal{N} \\
& a_{n p}-b_{p} \leq 0, \forall n \in \mathcal{N}, j \in \mathcal{S} \\
& \sum_{j \in S} b_{p}=P \\
& a_{n p}, b_{p} \in\{0,1\}, \forall n \in \mathcal{N}, j \in \mathcal{S}
\end{array}
$$

where $a_{n p}$ is a binary variable and is set to 1 if the demand $n$ is assigned to facility $p$, and it is zero otherwise. The location problems discussed above focus on providing QoS guarantees for the worst-case scenarios. Alternatively statistical measures can be considered minimising the average driving distance between any given number of charging stations. It is also possible to use median driving range as the statistical measure and the problem becomes $P$-median covering problem. Using similar notation, we formulate $P$-median problem as

$$
\begin{array}{ll}
\operatorname{minimize} & \sum_{n \in N} \sum_{p=1}^{P} w_{n} d_{n}\left(x_{p}, y_{p}\right) a_{n p} \\
\text { s.t. } & \sum_{p=1}^{P} a_{n p}=1, \forall n \in\{1, \ldots, N\} \\
& a_{n p} \in\{0,1\}, \forall n \in N, p \in\{1, \ldots, P\}
\end{array}
$$

An overview of discrete location problems are presented in Fig. 3.

\section{Continuous Network Models for Fast Charging Station}

In the previous section, we discuss that discrete location models are more suitable for slow charging stations as such 
chargers are deployed at candidate parking lots. Fast chargers, on the other hand, can be deployed anywhere in a network as long as there is supporting grid infrastructure. Hence, continuous network models are better suited for fast charging networks since they are smaller and can be located in any part of the network. The continuous location problems typically use $d$-dimensional real spaces. Distances between any two location is calculated according to Minkowski distances with the parameter $p$. For example, the $\ell_{p}$ distance between two separate points $\left(x_{i}, y_{i}\right)$ and $\left(x_{j}, y_{j}\right)$ with $i \neq j$ is defined as

$$
d_{i j}^{p}=\left[\left|x_{i}-x_{j}\right|^{p}+\left|y_{i}-y_{j}\right|^{p}\right]^{1 / p} .
$$

Most of the continuous models consider three values to $p$. For $p=1, \ell_{1}$ norm represents the Manhattan distance metric, that is,

$$
d_{i j}=\left|x_{i}-x_{j}\right|+\left|y_{i}-y_{j}\right| .
$$

For $p=2, \ell_{2}$ is the Euclidean distance and can be calculated as

$$
d_{i j}^{2}=\sqrt{\left|x_{i}-x_{j}\right|^{2}+\left|y_{i}-y_{j}\right|^{2}} .
$$

Finally, $p=\infty$ case corresponds to the Chebyshev metric and distance can be calculated as

$$
d_{i j}^{\infty}=\max \left\{\left|x_{i}-x_{j}\right|,\left|y_{i}-y_{j}\right|\right\} .
$$

In fast charging station location, the deployment principle is based on maximising the capture of the traffic flow between some origin and destinations. Flow-capturing location problems (FCLPs) present mathematical frameworks [23] and an illustrative example is presented in Fig. 4. In this example, two PEV charging stations are located on a route of possible 4 origin-destination pairs (O-D1, O-D2, O-D3, and O-D4). The serving region of two stations are shown circled and the traffic flows in O-D2 and O-D3 pairs can access to a charging station, while the other flows will not be captured by the located facilities. The FCLP problem only considers the case when a vehicle is captured or a region is covered. Such models does not consider service duration, vehicle types, or other related parameters. Hence, similar to discrete models, flow-capturing models do not address capacity of the charging nodes. Some application of flow capturing models include [24], [25], and [26].

Next, we proceed to discuss a toy example to show flow capturing models in a highway network. As shown in Figure 5 , an electric vehicle repeatedly travels 110 miles between the two cities, or an origin and destination pair. Suppose that PEV has a full battery at the origin and we aim to locate a fast charger along the O-D path. It is clear that the optimal charging location depends on the all-electric range (AER) of the vehicle. If the PEV has an AER of 220 miles (e.g., Tesla models) or more, then, the station can be sited at any location along the path since vehicle can complete its journey. As a second case, consider the case when the AER is 150 miles and the vehicle needs to recharge its battery either at destination $\mathrm{D}$ or some middle point on B-D path. Now, consider a third case in which the PEV has 85 miles of AER (a mainstream PEV model). In

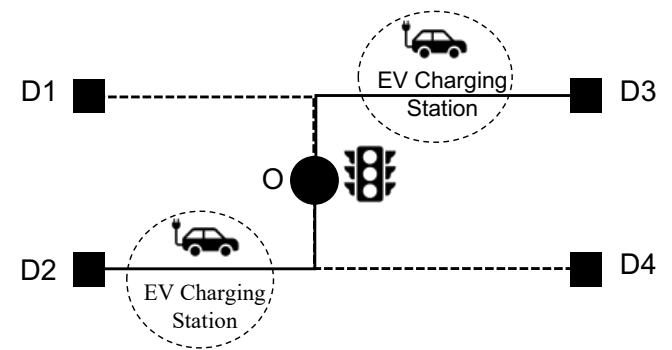

Fig. 4: Flow-capturing facility location problem for fast charging stations.

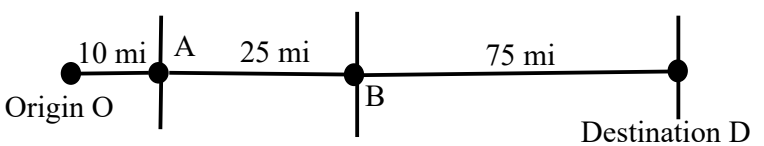

Fig. 5: Case study for fast charging stations.

this case, locating charging station exactly at nodes $0, \mathrm{D}, \mathrm{A}$, or B does not allow PEV to complete trip. If the model only allows for discrete locations, then there would be a need for at least two charging points (at nodes B and D). If stations are allowed to be deployed in middle points, then locating a station between B and D. More specifically, stations can be located at anywhere that is 25 to 42.5 miles away from point $\mathrm{D}$. This case study shows that continuous models lead to less number of charging points. A similar approach is used in [27] where optimal fast charging locations are investigated for California's highway network for different AER vehicles. In flow capturing models, the optimisation model takes a set-of O-D pairs and the associated traffic volumes as inputs. The model enumerates all different combinations of facility locations and aims to find the optimal one. Each combination is evaluated by the following typical mixed integer programming model [2],

$$
\begin{array}{ll}
\operatorname{maximize} & \sum_{q \in Q} f_{q} c_{q} \\
\text { s.t. } & \sum_{h \in H} u_{q h} v_{h} \geq c_{q}, \forall q \in Q \\
& e_{h n} a_{n} \geq v_{h}, \forall h \in H ; e \mid e_{h n}=1 \\
& \sum_{n \in N} a_{n}=P \\
& a_{n}, v_{h}, c_{q} \in\{0,1\}, \forall n \in N, \forall h \in H, q \in Q
\end{array}
$$

where, $Q$ is the set of all O-D pairs in a given region and $q$ is the index of 0-D pairs. Moreover, $f_{q}$ denotes PEV traffic demand along O-D path $q$, and $c_{q}$ is one if the traffic is captured and zero otherwise. $\mathrm{H}$ denotes set of all potential station combinations, $\mathrm{h}$ is the index of set $\mathrm{H}, e_{h n}$ is one if charging node $\mathrm{n}$ is in combination of $\mathrm{h}$, and zero otherwise.

\section{Probabilistic Location Methods}

Classical location theoretic approaches could be instrumental in providing coverage for PEV networks during the early phases of PEV integration. However, as the PEV sales increase, the capacity at one charging node may not be 
sufficient to provide good quality of service. In addition, there are a number of different factors affecting the design of charging stations. Depending on the location problem, factors enumerated below can be used as a part of objective function or take place in the constraint of the problem. Some of the most important ones are listed as below:

- Station Cost Factors: Components such as construction, operating, and maintenance costs. Construction cost contains physical equipments such as cabling, protection, chargers, and possibly storage units.

- Customer-related Factors: PEV driving and parking patterns, vehicle types, customer willingness to wait or defer service due to congestion.

- Technology-related Factors: Due to different standards and technologies, there are compatibility issues among charging stations PEVs (e.g., Tesla Super charger).

- Grid-related Factors: Available capacity on the supporting grid components such as substation, transmission lines, and local transformer.

In addition, charging station may employ storage units or renewable generators that can be used to support grid operators. Hence, there could be additional uncertainties in the supply side of the stations which may impacts grid operations [28]. In the next sections, we present an overview of stochastic models used in station location problems.

\section{A. Spatial Queuing}

In the field of urban operations research [29], spatial queuing models are widely used to locate distributed urban service systems such as police stations, ambulances, emergency repair, delivery vehicles, and transportation services. Larson [29] proposed a hypercube queueing model (HQM) which combines the queueing theory in facility location allocation problems given above. Spatial queueing is a descriptive model for designing spatially distributed server systems. The idea is to model each server (e.g., chargers) with a state space idle (0) or busy (1) for a given time instance.Then, entire charging network is represented by a multi dimensional system states. For a three server example, state (110) denotes the case when the first two servers are busy and the last one is idle. The solution of the model gives steady state probabilities of server utilisation, hence, associated performance measures such as average service rate, loss rate, waiting durations, etc. This model assumes that cities are divided into zones, which is already the case due to urban planning, and each charger is located in the middle of the zone. It is further assumed that $N$ servers are used to meet the demand originating at $M$ locations, where $N<M$. Therefore, one of the important topic of interest is to devise complex dispatch policies such as assigning servers to customers in a way to optimise networkwide performance. In spatial queuing models, location problems are solved by searching the best locations based on aforementioned performance metrics.

\section{B. Other Stochastic Models}

Due to the probabilistic nature of electric vehicle demand, stochastic modelling and optimisation have been widely adopted to locate and model charging networks. Queueing models enable designers to quantify important station performance parameters such as average or worst case waiting durations or probability of not getting a service. These metrics are used to determine the capacity of stations. Inspired from locating cellular networks near highways, [30] developed a station location framework using $\mathrm{M} / \mathrm{M} / \mathrm{s}$ queuing and fluid dynamics. In this mode, PEV arrivals and departures are assumed to be exponential and each station has s fast-chargers. Location and capacity problem of charging networks are further studies in Ref. [31] where customer demand is modelled with an $\mathrm{M} / \mathrm{M} / \mathrm{s}$ queueing model and customer convenience, measured with waiting times, are used as input for the optimisation model. Reference [32] considers both transportation and power grid constraints and solves placement problem by minimisation of station operation and investment cost. PEV demand is modelled by an M/M/s queue based on a given O-D pairs. In [33], authors developed a Bass diffusion model to forecast the PEV sales and determine the size of charging stations for the upcoming years. Furthermore, a queuing model was developed to optimise the location of charging facilities and an exhaustive search method was used to compare all cases and find minimum cost.

\section{Station Cost Minimisation}

A number of charging station location studies are studied from station cost minimisation perspective. In Ref. [34], station deployment cost is assumed to have three components, namely, charger cost, distribution network expansion cost, voltage regulation cost, and protection device upgrades. The location problem for urban areas is solved with a convex optimisation and subject to a number of constraints such as line current, power flow, voltage, and monetary limits. This type of approach is suitable for system operators and does not consider customer side dynamics. In addition, station siting is solved by hierarchical hybrid approaches that couples traffic models, both queuing and flow-capturing models, and cost minimisation. In [35], fast charging location problem is solved for highway networks. A similar approach is applied in [19], while each individual load is modelled by an $\mathrm{M} / \mathrm{M} / \mathrm{s} / \mathrm{s}$ meaning that there is no waiting space in charging stations.

\section{Station Capacity and Load Management}

While the main focus of this paper is to discuss deploying charging networks, locating enough number of charging points may be limited by power grid constraints such as line congestion and transformer loadings. Moreover, customer demand and vehicle distribution may not perfectly align. In this case, instead of increasing station capacity, customers can be dispatched to other neighbouring stations. Vehicle routing can be facilitated through financial incentives [36]. By employing similar financial incentives, PEV load can be 
deferred in time to off-peak hours to lower station capacity [37].

\section{CONCLUSIONS}

The success of electrification of transportation depends on the supporting grid infrastructure to provide necessary coverage and capacity to spatially distributed electric vehicles. In this paper, we presented a review of facility location theory for electric vehicle charging stations. We argued that classical operations research methods are suitable to address coverage issue, while capacity planning can be addressed by queueing models. Furthermore, for coverage models we showed that discrete network models are more appropriate for slow charging networks, while flow capturing models are suitable for fast charging stations. In the last part, we showed stochastic modelling approaches that captures the probabilistic nature of the PEV demand and station performance parameters such as average waiting time and customer rejection rates. Moreover, we discussed efforts on incentive based methods to manage loads at charging stations.

\section{REFERENCES}

[1] K. MacLellan and G. Faulconbridge, "Electric cars win? britain to ban new petrol and diesel cars from 2040," 2017.

[2] I. S. Bayram and A. Tajer, Plug-in electric vehicle grid integration. Artech House, 2017.

[3] C. d. M. Affonso and M. Kezunovic, "Probabilisitic sizing of pv generation on commercial parking lot with pevs to avoid transformer aging," in 2019 IEEE Milan PowerTech, June 2019, pp. 1-6.

[4] K. Clement-Nyns, E. Haesen, and J. Driesen, "The impact of charging plug-in hybrid electric vehicles on a residential distribution grid," IEEE Transactions on power systems, vol. 25, no. 1, pp. 371-380, 2009.

[5] Power networks navigate electric car challenge. [Online]. Available: https://www.ft.com/content/854526c8-30eb-11e7-9555-23ef563ecf9a

[6] I. S. Bayram, A. Tajer, M. Abdallah, and K. Qaraqe, "Capacity planning frameworks for electric vehicle charging stations with multiclass customers," IEEE Transactions on Smart Grid, vol. 6, no. 4, pp. 1934 1943, 2015.

[7] I. S. Bayram and M. Ismail, "A stochastic model for fast charging stations with energy storage systems," in 2019 IEEE Transportation Electrification Conference and Expo (ITEC), June 2019, pp. 1-5.

[8] T. for London, "https://tfl.gov.uk/modes/driving/low-emission-zone."

[9] C. S. ReVelle and H. A. Eiselt, "Location analysis: A synthesis and survey," European journal of operational research, vol. 165, no. 1, pp. $1-19,2005$.

[10] S. H. Owen and M. S. Daskin, "Strategic facility location: A review," European journal of operational research, vol. 111, no. 3, pp. 423-447, 1998.

[11] M. T. Melo, S. Nickel, and F. Saldanha-Da-Gama, "Facility location and supply chain management-a review," European journal of operational research, vol. 196, no. 2, pp. 401-412, 2009.

[12] M. S. Daskin, "What you should know about location modeling," Naval Research Logistics (NRL), vol. 55, no. 4, pp. 283-294, 2008.

[13] A. B. Arabani and R. Z. Farahani, "Facility location dynamics: An overview of classifications and applications," Computers \& Industrial Engineering, vol. 62, no. 1, pp. 408-420, 2012.

[14] G. O. Wesolowsky, "Dynamic facility location," Management Science, vol. 19 , no. 11, pp. 1241-1248, 1973.

[15] D. A. Giménez-Gaydou, A. S. Ribeiro, J. Gutiérrez, and A. P. Antunes, "Optimal location of battery electric vehicle charging stations in urban areas: A new approach," International Journal of Sustainable Transportation, vol. 10, no. 5, pp. 393-405, 2016.

[16] Z.-H. Zhu, Z.-Y. Gao, J.-F. Zheng, and H.-M. Du, "Charging station location problem of plug-in electric vehicles," Journal of Transport Geography, vol. 52, pp. 11-22, 2016.

[17] A. Y. Lam, Y.-W. Leung, and X. Chu, "Electric vehicle charging station placement: Formulation, complexity, and solutions," IEEE Transactions on Smart Grid, vol. 5, no. 6, pp. 2846-2856, 2014.
[18] S. Guo and H. Zhao, "Optimal site selection of electric vehicle charging station by using fuzzy topsis based on sustainability perspective," Applied Energy, vol. 158, pp. 390-402, 2015.

[19] C. Kong, R. Jovanovic, I. Bayram, and M. Devetsikiotis, "A hierarchical optimization model for a network of electric vehicle charging stations," Energies, vol. 10, no. 5, p. 675, 2017.

[20] F. Baouche, R. Billot, R. Trigui, and N.-E. El Faouzi, "Efficient allocation of electric vehicles charging stations: Optimization model and application to a dense urban network," IEEE Intelligent transportation systems magazine, vol. 6, no. 3, pp. 33-43, 2014.

[21] S. Srdic and S. Lukic, "Toward extreme fast charging: Challenges and opportunities in directly connecting to medium-voltage line," IEEE Electrification Magazine, vol. 7, no. 1, pp. 22-31, 2019.

[22] M. S. Daskin, Network and discrete location: models, algorithms, and applications. John Wiley \& Sons, 2011.

[23] T. D. Chen, K. M. Kockelman, and M. Khan, "Locating electric vehicle charging stations: Parking-based assignment method for seattle, washington," Transportation Research Record, vol. 2385, no. 1, pp. 2836, 2013.

[24] M. Cruz-Zambrano, C. Corchero, L. Igualada-Gonzalez, and V. Bernardo, "Optimal location of fast charging stations in barcelona: A flow-capturing approach," in 2013 10th International Conference on the European Energy Market (EEM), May 2013, pp. 1-6.

[25] R. Riemann, D. Z. Wang, and F. Busch, "Optimal location of wireless charging facilities for electric vehicles: flow-capturing location model with stochastic user equilibrium," Transportation Research Part C: Emerging Technologies, vol. 58, pp. 1-12, 2015.

[26] F. Wu and R. Sioshansi, "A stochastic flow-capturing model to optimize the location of fast-charging stations with uncertain electric vehicle flows," Transportation Research Part D: Transport and Environment, vol. 53, pp. 354-376, 2017.

[27] L. Zhang, B. Shaffer, T. Brown, and G. S. Samuelsen, "The optimization of dc fast charging deployment in california," Applied energy, vol. 157, pp. 111-122, 2015.

[28] T. Zhang, W. Chen, Z. Han, and Z. Cao, "Charging scheduling of electric vehicles with local renewable energy under uncertain electric vehicle arrival and grid power price," IEEE Transactions on Vehicular Technology, vol. 63, no. 6, pp. 2600-2612, July 2014.

[29] R. C. Larson and A. R. Odoni, Urban operations research, 1981, no. Monograph.

[30] S. Bae and A. Kwasinski, "Spatial and temporal model of electric vehicle charging demand," IEEE Transactions on Smart Grid, vol. 3, no. 1, pp. 394-403, 2011.

[31] T. Yi, X.-b. Cheng, H. Zheng, and J.-p. Liu, "Research on location and capacity optimization method for electric vehicle charging stations considering user's comprehensive satisfaction," Energies, vol. 12, no. 10, p. $1915,2019$.

[32] Y. Xiang, J. Liu, R. Li, F. Li, C. Gu, and S. Tang, "Economic planning of electric vehicle charging stations considering traffic constraints and load profile templates," Applied Energy, vol. 178, pp. 647-659, 2016.

[33] J. Ma and L. Zhang, "A deploying method for predicting the size and optimizing the location of an electric vehicle charging stations," Information, vol. 9, no. 7, p. 170, 2018.

[34] Q. Cui, Y. Weng, and C.-W. Tan, "Electric vehicle charging station placement method for urban areas," IEEE Transactions on Smart Grid, 2019.

[35] H. Zhang, S. J. Moura, Z. Hu, and Y. Song, "Pev fast-charging station siting and sizing on coupled transportation and power networks," IEEE Transactions on Smart Grid, vol. 9, no. 4, pp. 2595-2605, 2016.

[36] I. S. Bayram, G. Michailidis, and M. Devetsikiotis, "Unsplittable load balancing in a network of charging stations under qos guarantees," IEEE Transactions on Smart Grid, vol. 6, no. 3, pp. 1292-1302, 2014.

[37] I. S. Bayram, M. Ismail, M. Abdallah, K. Qaraqe, and E. Serpedin, "A pricing-based load shifting framework for ev fast charging stations," in 2014 IEEE International Conference on Smart Grid Communications (SmartGridComm). IEEE, 2014, pp. 680-685. 